Original Paper http://ajol.info/index.php/ijbcs http://indexmedicus.afro.who.int

\title{
Connaissances endogènes et perception du déficit hydrique liées à la culture du piment (Capsicum spp.) au Togo
}

\author{
Kwassi Kporliawornou LEDI ${ }^{1 *}$, Komi ODAH ${ }^{1}$, Hodabalo KAMOU ${ }^{2}$, \\ Atalaèsso BOKOBANA ${ }^{1}$, Lidaou EGBARE ${ }^{1}$, Sêminhinva AKPAVI² et Koffi TOZO ${ }^{1}$ \\ ${ }^{1}$ Laboratoire de Physiologie et de Biotechnologie Végétales, Faculté des Sciences, Université de Lomé, BP \\ 1515, Lomé-Togo. \\ ${ }^{2}$ Laboratoire de Botanique et Ecologie Végétales, Université de Lomé, BP 1515, Lomé, Togo. \\ *Auteur correspondant, Courriel : ledikwassi380@gmail.com; Tel : 0022890238027.
}

\section{RESUME}

Au Togo, la production du piment (Capsicum spp.), légumineuse prisée, reste encore très faible. Afin d'aider à sa promotion, une enquête a été menée dans 20 localités, pour évaluer les contraintes qui y sont liées, la perception paysanne du déficit hydrique et les pratiques endogènes de gestion de l'eau. Au total, 28 contraintes ont été répertoriées dont la plus citée est le déficit hydrique (94,74\%). La corrélation entre les localités et les contraintes citées a permis de définir trois groupes de localités dont deux géographiquement homogènes. La région centrale, avec le plus grand nombre de contraintes, connaît également les plus longues durées de poches de sécheresse en pleine saison pluvieuse $(2,4 \pm 0,75$ semaines). En périodes de manque d'eau, les producteurs adoptent diverses pratiques endogènes consommatrices d'eau (irrigation, arrosage) et/ou conservatrices d'humidité (paillage, agriculture sur berge). Aussi, 34 cultivars, sous réserve de synonymie, ont été identifiés, répartis en six groupes suivant une classification ascendante hiérarchique basée sur la forme des fruits. Le cultivar Gboyébéssé est le plus prisé avec un indice de valeur d'importance de 1,22. Ces résultats pourront permettre de mieux planifier les plans d'action des différents acteurs de la filière piment pour une production optimale.

(C) 2020 International Formulae Group. All rights reserved.

Mots clés : Légumes, stress hydrique, pratiques endogènes, contraintes de culture, Togo.

\section{Endogenous knowledges and perception of water deficit related to chilli (Capsicum spp.) cultivation of in Togo}

\begin{abstract}
In Togo, the production of chili pepper (Capsicum spp.), estimated vegetable, is still very low. In order to help its promotion, a survey was conducted in 20 areas, to assess the related constraints, the peasant perception of water deficit and the endogenous practices of water management. A total of 28 constraints were identified and the most cited is water deficit (94.74\%). Three groups of areas (of which two are geographically homogeneous) emerged after a correlation between areas and constraints. The central region, with the highest
\end{abstract}


number of constraints cited, also has the longest dry pockets in the wet season ( $2.4 \pm 0.75$ weeks). In response to periods of lack of water, producers adopt various endogenous water-consuming practices (irrigation, watering) and/or humidity-conserving practices (mulching, riverbank farming). Also, the study identified 34 cultivars if no synonymy, divided into six groups according to an ascending hierarchical classification and based on the pomological characteristics of the fruit. Cultivar Gboyébéssé is the most popular cultivar with a value index of 1.22. These results can be the basis for better action plan of the various stakeholders in the chilli pepper sector for an optimal production.

(C) 2020 International Formulae Group. All rights reserved.

Keywords: Vegetables, water stress, endogenous practices, cultural constraints, Togo.

\section{INTRODUCTION}

Le piment (Capsicum spp.) est une espèce légumière largement cultivée dans le monde. Il est consommé sous forme fraiche, sèche, entière ou en poudre (Tano et al., 2008 ; Reifschneider et al., 2009). Il est riche en protéines, lipides, glucides, fibres, sels minéraux $(\mathrm{Ca}, \mathrm{P}, \mathrm{Fe})$ et en vitamines $\mathrm{A}, \mathrm{D}, \mathrm{E}$, C, K, B2 (Xing et al., 2011; Tilahun et al., 2013). C'est un produit de base de la cuisine chez de nombreux groupes ethniques en Asie, en Amérique Latine et en Afrique (Kollmannsberger et al. 2011) pour son action stimulante qui rehausse la saveur des aliments, en augmentant la salivation et la sensation de chaleur dans la bouche (Kouassi et KoffiNevry, 2012). Les oléorésines qui sont extraites des fruits secs sont utilisées dans la production de colorants de divers produits (Meghvansi et al., 2010). Il est aussi utilisé comme bio pesticide dans des programmes de lutte intégrée (Simo et al., 2019).

Connus aussi comme plante médicinale, les fruits de Capsicum sont employés en médecine traditionnelle pour leurs propriétés antimicrobiennes dues aux métabolites secondaires qu'ils contiennent (Hervert-Hernández et al., 2010). Le piment possède également des propriétés antioxydantes, antimutagènes, hypocholestérolémiques et immunosuppressives (Wahyuni et al., 2013).

Sur le plan économique, le piment reste, au niveau mondial, l'une des épices qui génèrent des revenus énormes aux producteurs, avec des prix variant de 95 à 1.300 FCFA par kilogramme (pour les piments frais) et de 1.200 à 6.000 FCFA par kilogramme (pour les piments secs). Il contribue ainsi à l'amélioration des conditions de vie, notamment celle des femmes en milieu rural (Kwon et al., 2006). Malheureusement, malgré la possibilité de le cultiver dans la plupart des régions du pays, les rendements du piment au Togo n'atteignent pas encore ceux d'autres pays d'Afrique de l'ouest à l'instar du Nigeria et du Ghana et ne cessent de chuter d'année en année. Pour exemple, la production nationale de piment doux et épicé entre 2008 et 2018 a connu une très faible évolution, passant de 7.750 tonnes en 2008 à 8.130 tonnes en 2018 soit une augmentation de 4,7\% (FAO, 2019). Ces faibles rendements seraient dus à de nombreuses contraintes biotiques (insectes ravageurs, champignons, virus, bactéries...) et abiotiques dont le déficit hydrique serait la principale, eu égard aux sérieux changements du régime pluviométrique que connaît le pays ces dernières décennies (Lemou, 2008 ; Badameli et Dubreuil, 2010 ; Adewi et al., 2010).

Dans ce contexte, cette étude vise l'évaluation des contraintes liées à la culture $\mathrm{du}$ piment, de la perception paysanne du déficit hydrique et des pratiques endogènes de gestion de l'eau, afin de promouvoir cette culture et d'atténuer les effets de la pauvreté au Togo et à terme contribuer à rehausser l'économie du pays. 


\section{MATERIEL ET MÉTHODES}

\section{Choix des localités prospectées et collecte des données}

L'étude a été menée dans 20 localités (Figure 1) choisies sur la base des travaux de l'Institut Togolais de Recherche Agronomique (ITRA), dans le cadre du projet PPAO (Programme de Productivité Agricole en Afrique de l'Ouest) mis en œuvre au Togo en juillet 2015. Cette étude visait à vulgariser des variétés introduites de piment au Togo et les localités objet de l'enquête sont réputées dans la culture du piment.

Pour la conduite de cette enquête, les ménages ont été choisis au hasard dans les localités prospectées. Ainsi, les producteurs de piments en champs et/ou en maraîchers ont été ciblés par l'enquête. Toutes les couches sociales (hommes, femmes, jeunes, personnes âgées) ont été interrogées avec un total de 100 personnes questionnées. L'approche méthodologique se base sur celle de Loko, 2013. Elle est à la fois qualitative et quantitative : entretiens, focus groupe et des enquêtes de terrain à l'aide d'un questionnaire structuré. Les discussions ont été conduites de façon ouverte et sans limitation de temps, afin de permettre aux producteurs d'exprimer librement leurs connaissances conformément à la méthode de Dansi et al., 2010. Les données recueillies concernent essentiellement, les contraintes liées à la culture du piment, la durée des poches de sécheresse en pleine saison des pluies, les pratiques culturales adoptées en situation de manque d'eau, l'importance économique des cultivars. Pour les contraintes biotiques, des photos tirées de la bibliographie ont préalablement été imprimées, afin de permettre aux producteurs de reconnaître les effets des agents biologiques considérés.

\section{Traitement des données}

Les différents cultivars répertoriés ont été classés en fonction de trois critères: la résistance à la sècheresse, la prédominance et l'importance économique. La méthode de choix multicritère a permis, sur la base de ces critères, de classer par région économique ces cultivars. Pour chaque critère, le cultivar a un indice de valeur que nous avons multiplié par le poids du critère. La somme pondérée de l'indice de valeur de chaque cultivar par le poids du critère nous donne la valeur du cultivar pour ces trois critères combinés (Tableau 1). Afin d'évaluer l'importance réelle des cultivars répertoriés, il a été déterminé leur indice de valeur d'importance (IVI) qui fait une corrélation entre leur superficie emblavée et leur prédominance, suivant la formule :

\section{$I V I=$ FrQRpd + $S^{2} A c / S^{2} T+F r Q \_L o c$. FrQ_Rpd}

IVI = Indice de valeur d'importance.

FrQ_Rpd (Fréquence des répondants) = nombre de personnes qui cultivent le cultivar/sur le nombre de personnes qui cultivent le piment.

$\mathbf{S}^{2} \mathbf{A c} / \mathbf{S}^{2} \mathbf{T}=$ La superficie du cultivar considéré sur la superficie totale réservée à la culture du piment. FrQ_Loc (Fréquence des localités) $=$ nombre de localités dans lesquelles le cultivar est cultivé sur le nombre total de localités enquêtées.

\section{Analyse des données de l'enquête}

Les données obtenues ont été soumises à des analyses statistiques descriptives et multivariées à l'aide des logiciels Xlstat 2008 et Minitab 16. Elles ont également été transcrites sur Excel et les résultats sont traduits sous forme de graphe et de dendrogramme. 


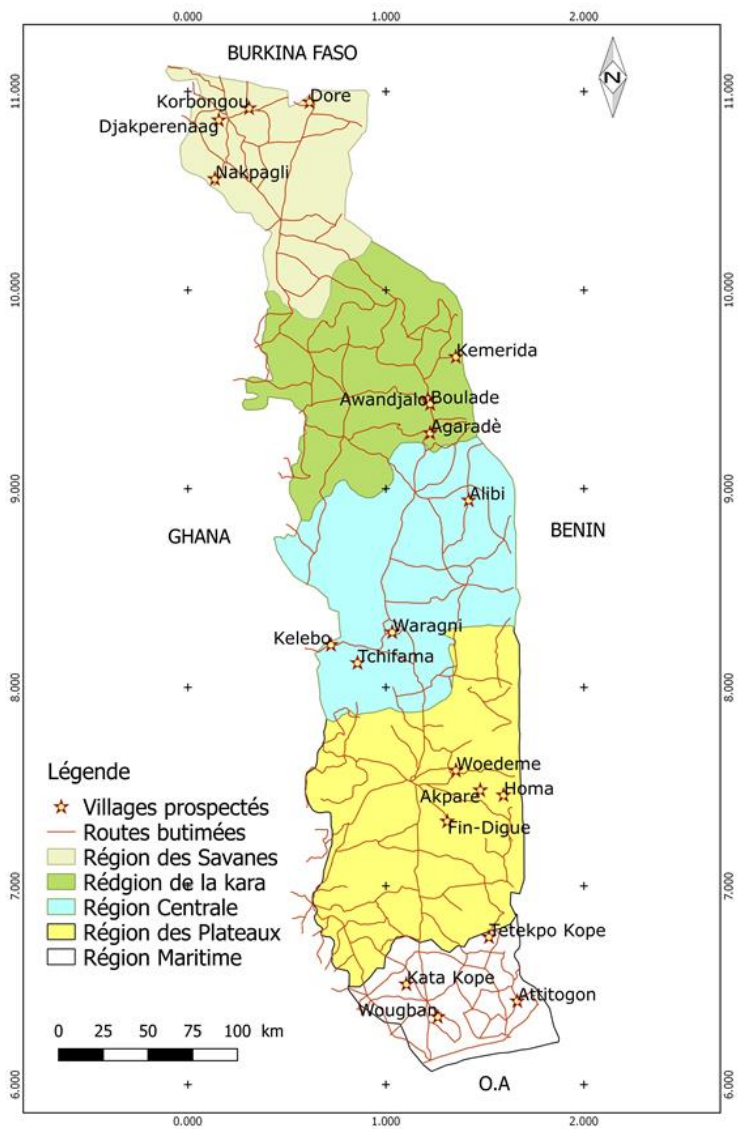

Figure 1 : Carte montrant les localités prospectées.

Tableau 1 : Méthode de choix par multicritère.

\begin{tabular}{|c|c|c|c|c|}
\hline & $\begin{array}{l}\text { Résistance à la } \\
\text { sècheresse }\end{array}$ & Prédominance & Importance économique & Valeur du cultivar \\
\hline $\begin{array}{l}\text { Poids des } \\
\text { critères }\end{array}$ & $\begin{array}{l}\text { W'a } \\
W^{\prime} a=W a / \\
(W a+W b+W c)\end{array}$ & $\begin{array}{l}\mathrm{W}^{\prime} \mathrm{b} \\
\mathrm{W}^{\prime} \mathrm{b}=\mathrm{Wb} /(\mathrm{Wa}+ \\
\mathrm{Wb}+\mathrm{Wc})\end{array}$ & $\begin{array}{l}\text { W'c } \\
\mathrm{W}^{\prime} \mathrm{c}=\mathrm{Wc} /(\mathrm{Wa}+\mathrm{Wb}+\mathrm{Wc})\end{array}$ & $W^{\prime} a+W^{\prime} b+W^{\prime} c=1$ \\
\hline Cul 1 & V1a & V1b & V1c & $\begin{array}{l}\text { Z1= (V1a.W'a) + (V1b.W'b) } \\
+(\text { V1c.W'c) }\end{array}$ \\
\hline $\mathrm{Cul} 2$ & V2a & $\mathrm{V} 2 \mathrm{~b}$ & $\mathrm{~V} 2 \mathrm{c}$ & $\begin{array}{l}\mathrm{Z} 2=(\mathrm{V} 2 \mathrm{a} \cdot \mathrm{Wa})+(\mathrm{V} 2 \mathrm{~b} \cdot \mathrm{Wb}) \\
+(\mathrm{V} 2 \mathrm{c} \cdot \mathrm{Wc})\end{array}$ \\
\hline • & $\cdot$ & • & $\cdot$ & $\cdot$ \\
\hline . & . & . & . & $\cdot$ \\
\hline Cul 6 & V6a & V6b & V6c & $\begin{array}{l}\mathrm{Z6}=(\mathrm{V} 6 \mathrm{a} \cdot \mathrm{Wa})+(\mathrm{V} 6 \mathrm{~b} \cdot \mathrm{Wb})+ \\
(\mathrm{V} 6 \mathrm{c} \cdot \mathrm{Wc})\end{array}$ \\
\hline
\end{tabular}

Cul : cultivar; Wa : proportion de personnes affirmant qu'il existe un cultivar qui résiste aux longues périodes de sécheresse dans la localité ; Wb : proportion de personnes affirmant ne pas délaisser la culture du piment au profit d'autres cultures dans la région; Wc: Proportion de personnes affirmant que la culture du piment constitue leur principale source de revenue ; Via : proportion de personnes ayant cité le cultivar i comme résistant à la sècheresse parmi les personnes qui affirment qu'il existe un cultivar qui résiste aux longues périodes de sécheresse; Vib : proportion de personnes ayant cité le cultivar i comme étant produit dans le milieu et étant le plus cultivé parmi les personnes qui affirment ne pas délaisser la culture du piment au profit d'autres cultures dans la région; Vic : proportion de personne ayant cité le cultivar i comme étant le plus cultivé et qui constitue sa première source de revenue parmi les personnes qui affirme que la culture du piment constitue leur principale source de revenue. Le classement se fait en comparant les $\mathrm{Zi}$. 


\section{RÉSULTATS}

\section{Contraintes liées à la production du piment au Togo}

$\mathrm{Au}$ total, 28 contraintes ont été recensées sur toute l'étendue du territoire (Figure 2). Les contraintes majeures sont : le déficit hydrique $(94,74 \%)$, la pauvreté du sol $(89,47 \%)$, la conservation difficile des fruits $(84,21 \%)$ et le flétrissement bactérien $(64,82 \%)$. Les contraintes les moins citées sont le manque de main d'œuvre, l'absence de marché de vente, la pourriture molle des fruits et le retard de croissance de certains cultivars (5,26\% respectivement).

Par ailleurs, les contraintes répertoriées varient d'une région économique à une autre et n'ont pas partout la même importance. Une analyse des résultats par région révèle une disparité du nombre et des types de contraintes (Figure 3). La région centrale connaît plus de contraintes liées à la culture du piment, soit 18 contraintes citées. Si le déficit hydrique est cité comme contrainte majeure dans les régions des savanes $(100 \%)$, des plateaux $(100 \%)$ et maritime $(85,71 \%)$, il n'en est pas de même dans les régions centrales et de la Kara où sont plutôt redoutées les attaques d'insectes $(87,50 \%$ et $88,89 \%$ respectivement).

\section{Corrélation entre les contraintes majeures et les localités prospectées mises en évidence par l'ACP}

L'étude de la corrélation entre les contraintes majeures et les localités prospectées a été évaluée grâce à une analyse en composantes principales (ACP). Les résultats ont permis de différencier trois groupes de localités dont deux sont géographiquement homogènes (Figure 4, Tableau 2). Il s'agit du groupe I qui regroupe des localités de la région de la Kara (Bouladè, Awandjélo et Kémérida), du groupe II qui regroupe des localités de la région des plateaux (Kelebo, Waragni et Tchifama). Le groupe III par contre est géographiquement disparate avec des localités aussi bien des régions maritimes, des plateaux que des savanes (Tétékpoé Kopé, Attitongon, Homa, Akparé, Fin digue, Wougba, Djakperenaag).

\section{Perception paysanne du déficit hydrique et pratiques endogènes}

A la suite des enquêtes menées il a pu être déterminé que la région centrale connait la plus longue durée moyenne de poches de sécheresse, soit 2,4 semaines contre 1,9 semaine ; la plus faible durée, dans la région des savanes (Tableau 3).

Lors de ces périodes de manque d'eau, les paysans adoptent diverses pratiques endogènes d'adaptation (Figure 5):

- Arrosage avec de l'eau des cours d'eau ou des retenues d'eau avoisinantes (dans les zones ayant un réseau hydrographique important),

- Agriculture sur berge pour profiter de l'humidité apparente du sol (plus courante dans la région des savanes où le réseau hydrographique est moins important et la sécheresse plus prononcée),

- Paillage avec utilisation de feuilles de palmiers pour la plupart du temps en vue de conserver l'humidité souvent pour les pépinières de piment (pratique dans toutes les zones),

- Irrigation avec utilisation de méthodes parfois améliorées (dispositif de tuyauteries installées dans les sillons des champs et en association avec des retenues d'eau aménagées, pratique plus moderne que les précédentes).

D'autres, par contre, abandonnent toute activité agricole en attendant la bonne saison (Tableau 3).

Classification ascendante hiérarchique des cultivars de piment sur la base des caractères pomologiques du fruit

Les cultivars répertoriés présentent diverses formes de fruits et peuvent être ainsi regroupés à travers une classification ascendante hiérarchique (CAH) (Figure 6). Cependant, une analyse statistique permet de les rapprocher. Au seuil de dissimilarité de 1,6 en se basant sur la couleur du tégument et la forme du fruit, on distingue six groupes de cultivars dont quelques échantillons sont illustrés à la Figure 7 : 
- Le groupe 1: Cultivars aux fruits ronds, moyens, de couleur verte vive au bout aplati (cas de Djakami).

- Le groupe 2: Cultivars aux fruits allongés mais plus aplatis, jaunes pâles à jaunes fluor (cas ICRAD I).

- Le groupe 3: Cultivars aux fruits ronds, verts vifs à verts pâles et au bout aplati (cas de Tongoor).

- Le groupe 4 : Cultivars aux fruits effilés de couleur vert - foncé, au bout pointu (droit ou courbé) (cas de Adonlobor).

- Le groupe 5 : Cultivars aux fruits rouges, allongés mais courts et à bout arrondi (cas Gobi).
- Le groupe 6. Cultivars aux fruits effilés et longs de couleur rouge et au bout arrondi (cas de Lèguèlèguè).

\section{Indice de valeur d'importance des cultivars de piment inventoriés}

Le Tableau 4 présente l'indice de valeur d'importance des différents cultivars de piment. De l'analyse de ce tableau, il en ressort que le cultivar Gboyébéssé a l'indice de valeur d'importance le plus élevé, suivi de Adonlobo, poivron, Tongor soient des indices de valeur d'importance respectivement de 1,$22 ; 0,28 ; 0,25$ et 0,24 .

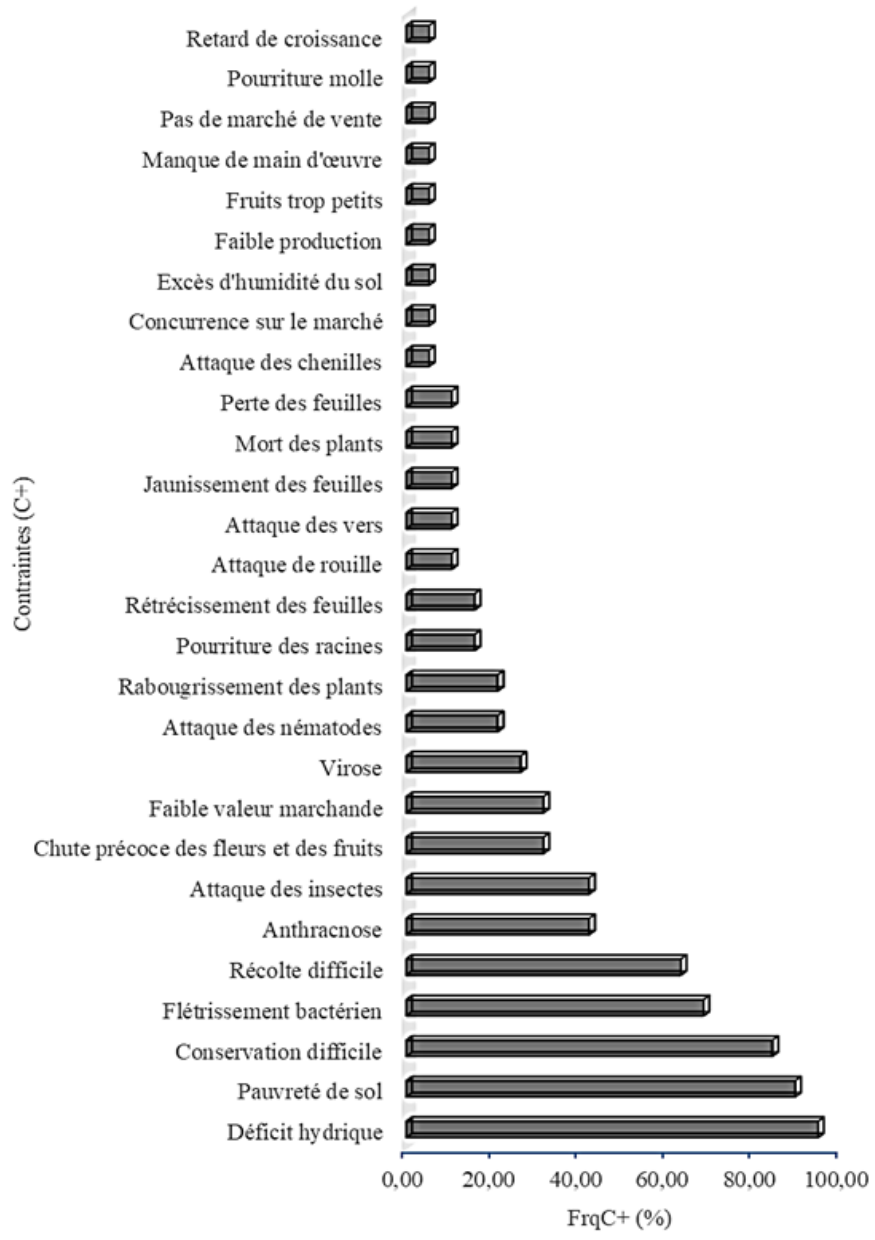

Figure 2 : Contraintes liées à la culture du piment dans l'ensemble des localités prospectées. 


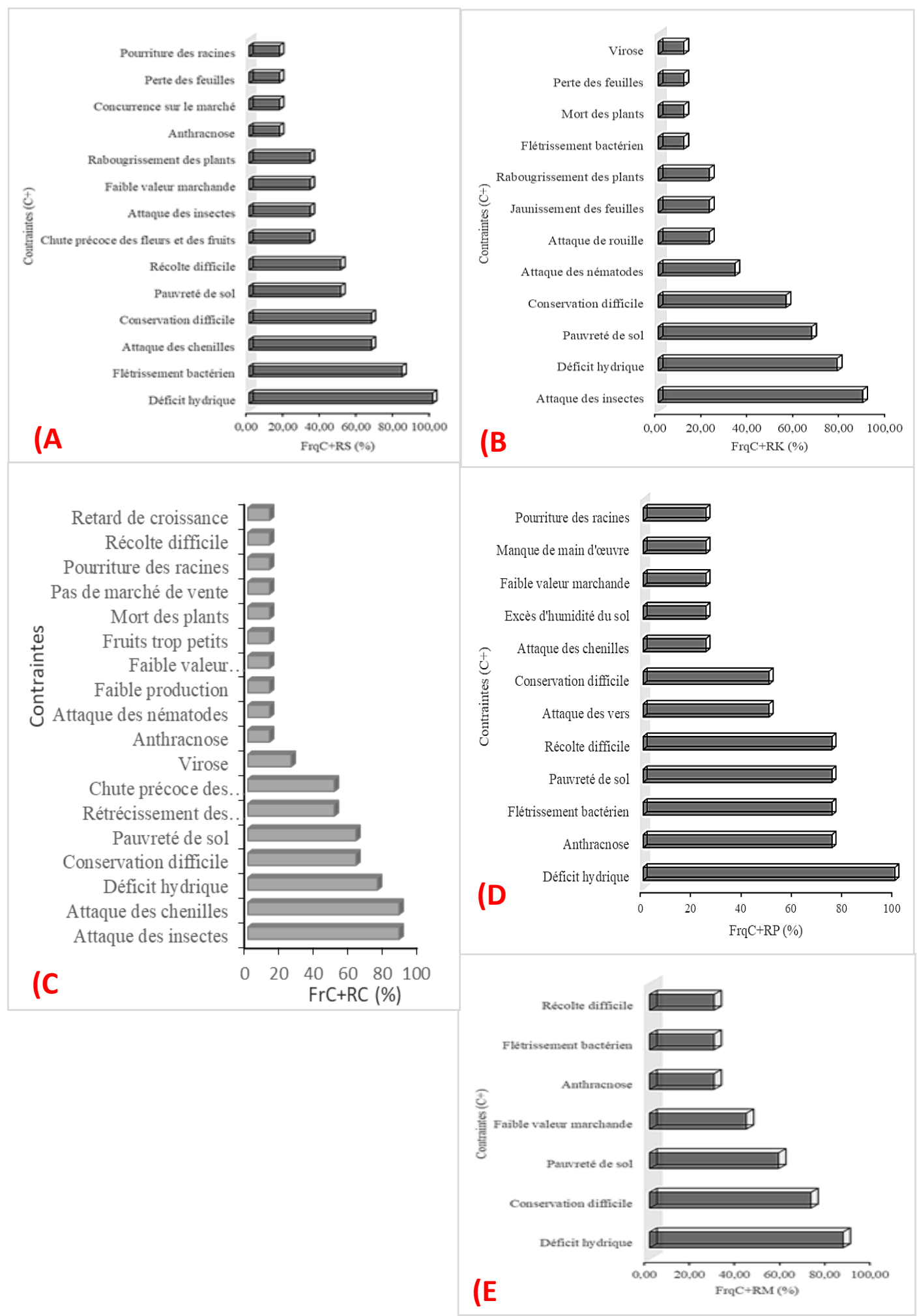

Figure 3 : Contraintes liées à la culture du piment dans les localités prospectées par région économique. (A) Région des savanes ; (B) Région de la Kara ; (C) Région centrale ; (D) Région des plateaux ; (E) Région maritime. 


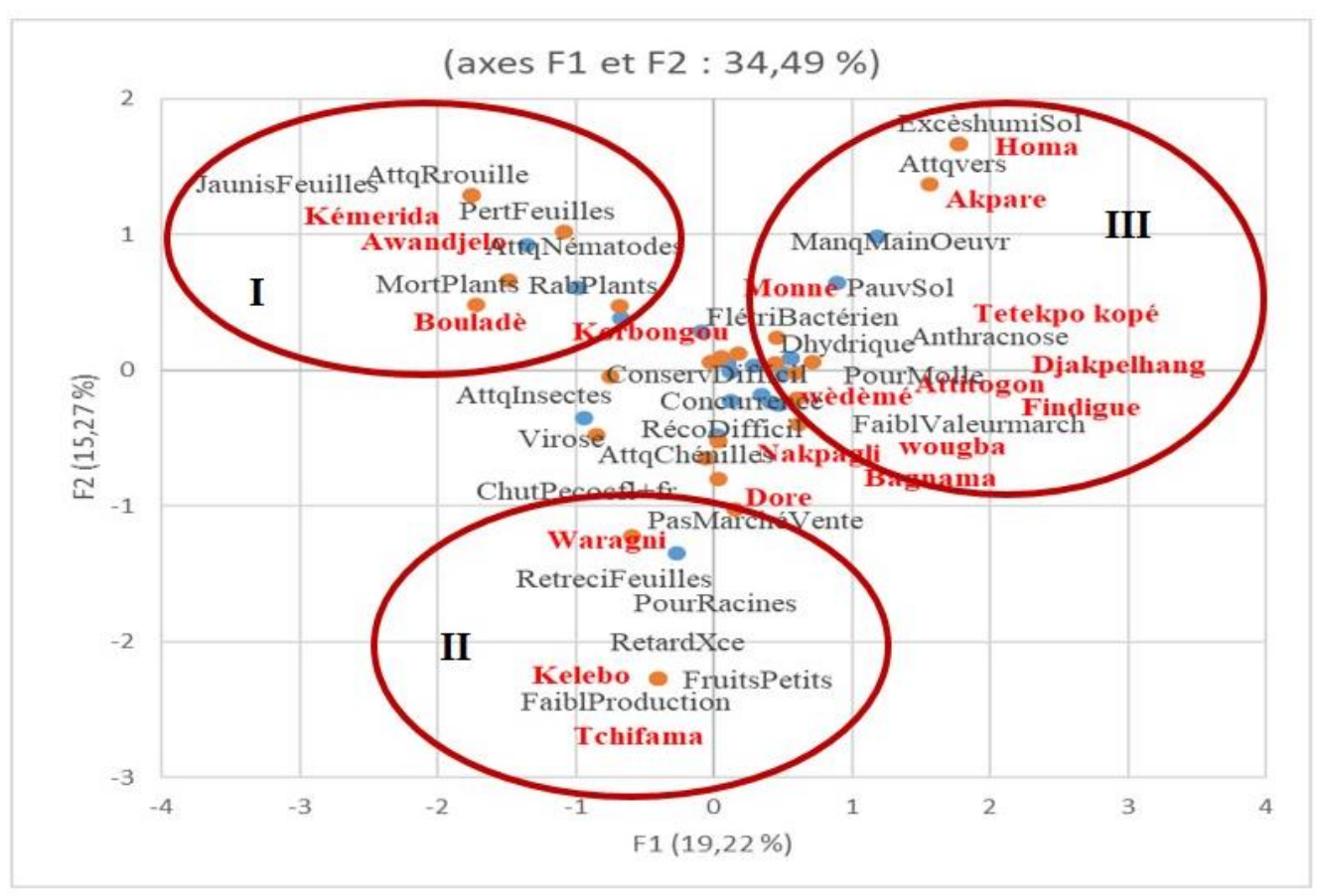

Figure 4 : Corrélation entre les contraintes et les localités enquêtées.

Tableau 2: Classification des localités ayant les mêmes contraintes majeures.

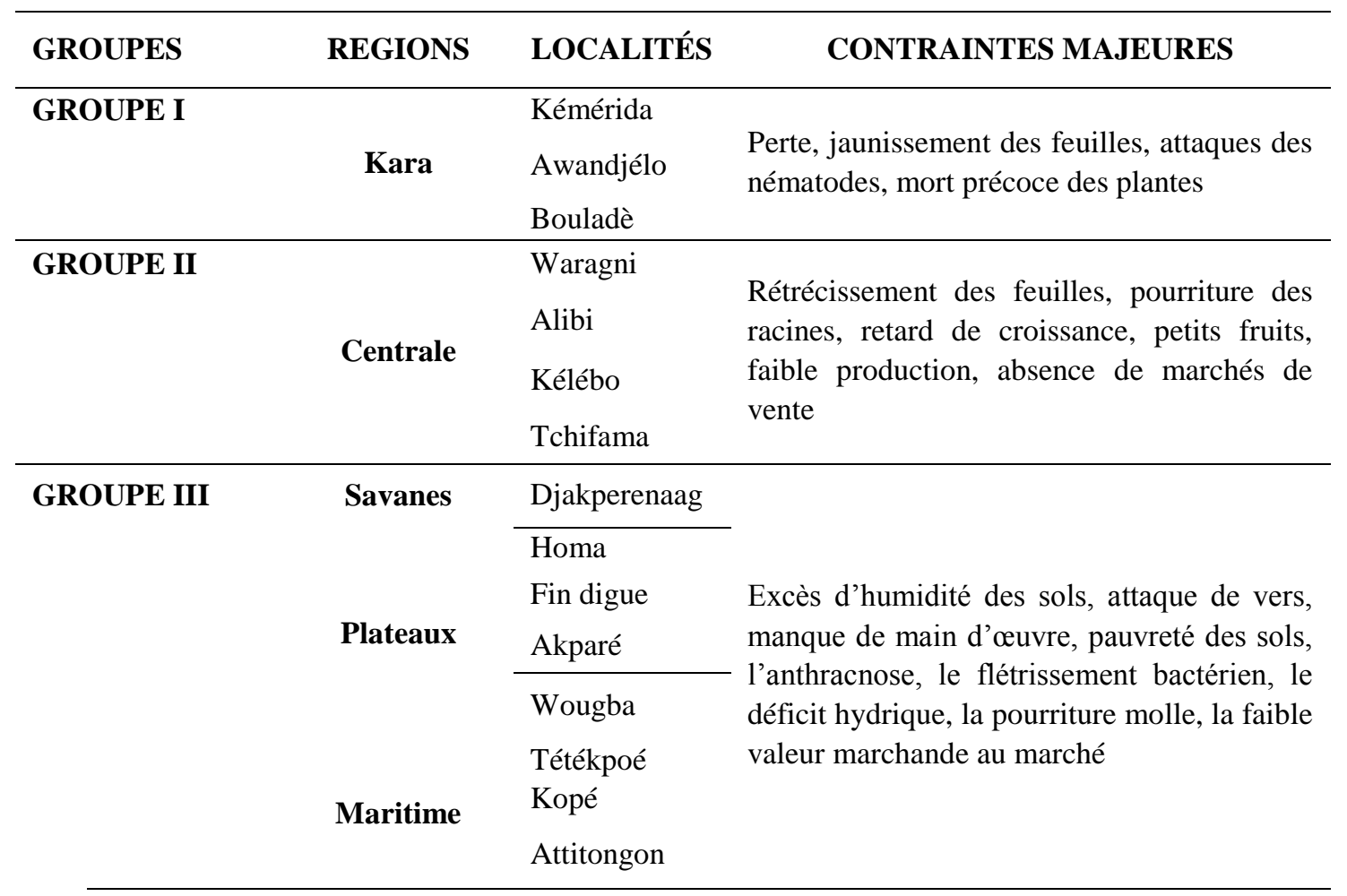


Tableau 3 : Durée moyenne des poches de sécheresse par région.

\begin{tabular}{|c|c|c|}
\hline Régions & $\begin{array}{l}\text { Durée moyenne (semaines) } \\
\text { des poches de sècheresse }\end{array}$ & Pratiques endogènes d'adaptation \\
\hline Savanes & $1,90 \pm 0.92$ & $\begin{array}{l}\text { - Irrigation : } 59 \% \\
\text { - Arrosage : } 31 \% \\
\text { - Agriculture sur berge : } 10 \%\end{array}$ \\
\hline Kara & $2,17 \pm 0.71$ & $\begin{array}{l}\text { - Agriculture sur berge : } 52 \% \\
\text { - Irrigation : } 17 \% \\
\text { - Paillage : } 17 \% \\
\text { - Cessation : } 11 \% \\
\text { - Arrosage : } 3 \%\end{array}$ \\
\hline Centrale & $2,4 \pm 0.75$ & $\begin{array}{l}\text { - Irrigation : } 79 \% \\
\text { - Cessation : } 21 \%\end{array}$ \\
\hline Plateaux & $2,28 \pm 0.84$ & $\begin{array}{l}\text { - Irrigation : } 46 \% \\
\text { - Agriculture sur berge : } 19 \% \\
\text { - Paillage : } 14 \% \\
\text { - Arrosage : } 11 \% \\
\text { - Cessation : } 10 \%\end{array}$ \\
\hline Maritime & $2 \pm 0.81$ & $\begin{array}{l}\text { - Cessation : } 80 \% \\
\text { - Paillage : } 20 \%\end{array}$ \\
\hline
\end{tabular}
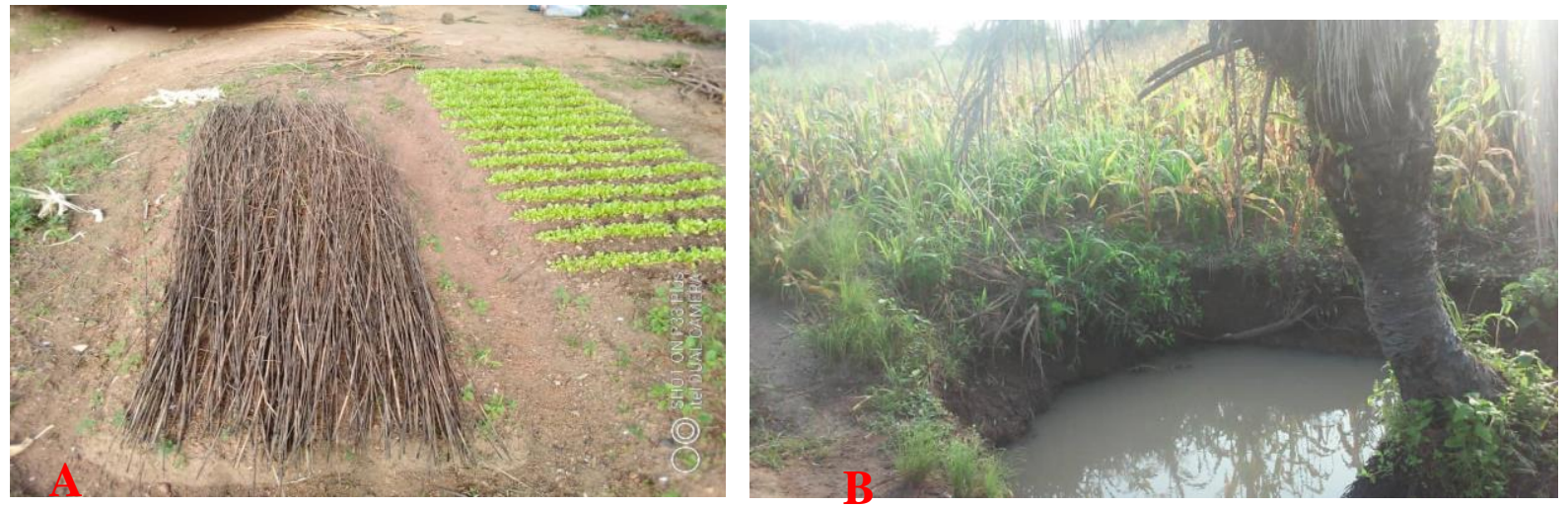

$*$

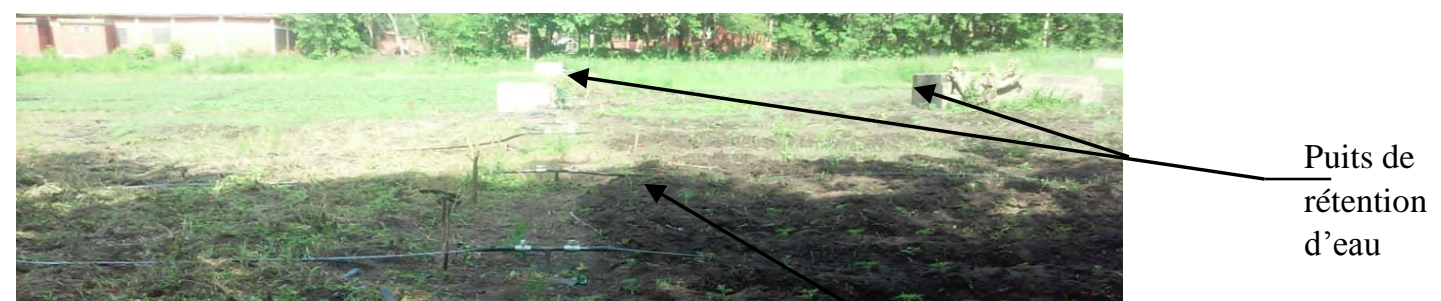

Tuyauterie d'arrosage

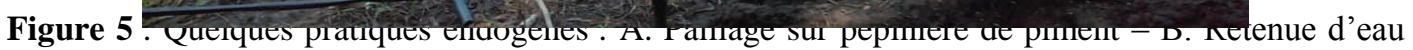
dans un champ (trou creusé pour recueillir l'eau de pluie) - C. Installation de tuyauteries d'arrosage dans les sillons avec un point de retenue d'eau (puits) 


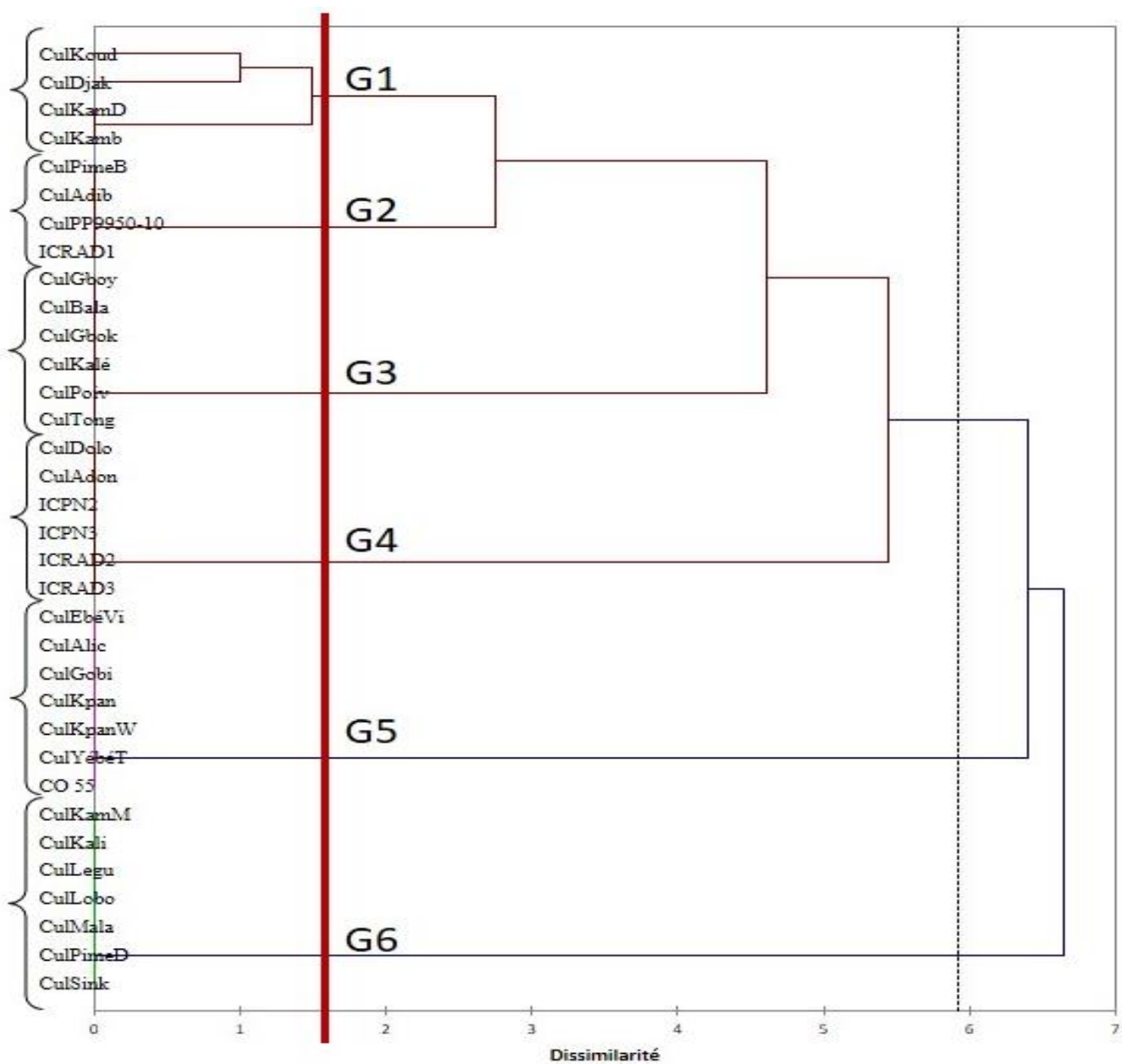

Figure 6 : Classification ascendante hiérarchique des cultivars de piment sur la base des caractères pomologiques du fruit.
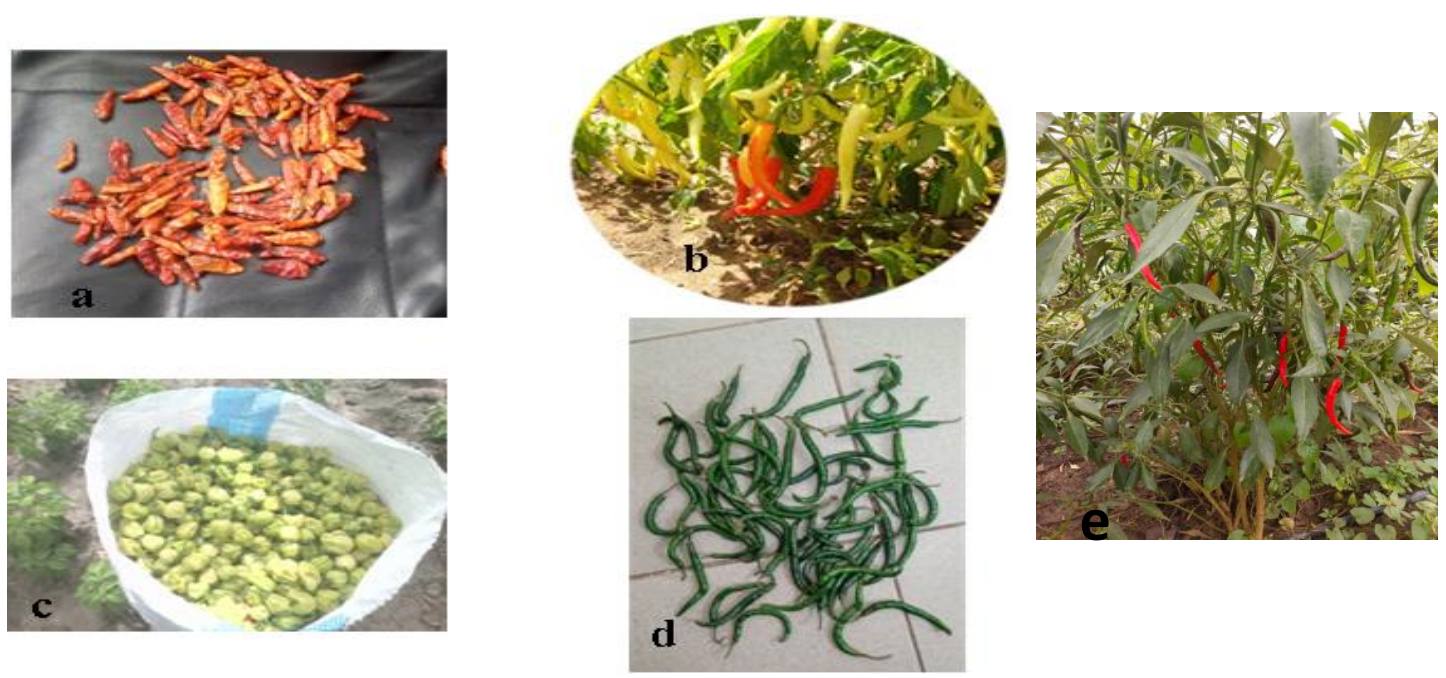

Figure 7 : Quelques cultivars représentatifs des différentes classes de piment inventoriées (a) Gobi, (b) ICRAD I, (c) Tongor, (d) Adonlobor. (e) Lèguèlèguè. 
Tableau 4 : Indice de valeur d'importance des cultivars de piment inventoriés.

\begin{tabular}{|c|c|c|c|c|c|c|c|}
\hline Cultivars & N_Rpdts & FrQRpd & $\mathbf{S}^{2}$ & $\mathbf{S}^{2} \mathbf{A c} / \mathbf{S}^{2} \mathbf{T}$ & N_LOc & FrQLoc & IVI \\
\hline Gboyébéssé & 81 & 0,30 & 223421,87 & 0,333 & 17 & 0,59 & 1,22 \\
\hline Adonlobo & 20 & 0,07 & 45625 & 0,068 & 4 & 0,14 & 0,28 \\
\hline Poivron & 20 & 0,07 & 24281,25 & 0,036 & 4 & 0,14 & 0,25 \\
\hline Tongor & 16 & 0,06 & 25625 & 0,038 & 4 & 0,14 & 0,24 \\
\hline Lenguélengué & 8 & 0,03 & 10625 & 0,016 & 5 & 0,17 & 0,22 \\
\hline Gobi & 24 & 0,09 & 10000 & 0,015 & 3 & 0,10 & 0,21 \\
\hline Kpenzoudonga & 18 & 0,07 & 19375 & 0,029 & 3 & 0,10 & 0,20 \\
\hline Lobolobo & 8 & 0,03 & 16250 & 0,024 & 2 & 0,07 & 0,12 \\
\hline ICPN3 & 6 & 0,02 & 36875 & 0,055 & 1 & 0,03 & 0,11 \\
\hline PN 9955-10 & 6 & 0,02 & 36250 & 0,054 & 1 & 0,03 & 0,11 \\
\hline Kambouga & 7 & 0,03 & 7343,75 & 0,011 & 2 & 0,07 & 0,11 \\
\hline Sinkpodji & 4 & 0,01 & 1250 & 0,002 & 2 & 0,07 & 0,09 \\
\hline Koudjadakpè & 2 & 0,01 & 1250 & 0,002 & 2 & 0,07 & 0,08 \\
\hline Kpenzou wiloor & 2 & 0,01 & 1250 & 0,002 & 2 & 0,07 & 0,08 \\
\hline $\begin{array}{l}\text { Tchangai } \\
\text { Koumonka }\end{array}$ & 2 & 0,01 & 1250 & 0,002 & 2 & 0,07 & 0,08 \\
\hline Kam djé & 2 & 0,01 & 0,1 & 0,000 & 2 & 0,07 & 0,08 \\
\hline $\begin{array}{l}\text { Kamme } \\
\text { moanne }\end{array}$ & 1 & 0,00 & 625 & 0,001 & 2 & 0,07 & 0,07 \\
\hline Balankong & 2 & 0,01 & 20000 & 0,030 & 1 & 0,03 & 0,07 \\
\hline Malanville & 6 & 0,02 & 5625 & 0,008 & 1 & 0,03 & 0,07 \\
\hline $\begin{array}{l}\text { Yébéssévi } \\
\text { toukoui }\end{array}$ & 5 & 0,02 & 5625 & 0,008 & 1 & 0,03 & 0,06 \\
\hline Dibolo & 1 & 0,00 & 10000 & 0,015 & 1 & 0,03 & 0,05 \\
\hline Kam mond & 3 & 0,01 & 5000 & 0,007 & 1 & 0,03 & 0,05 \\
\hline Dolobo & 3 & 0,01 & 2500 & 0,004 & 1 & 0,03 & 0,05 \\
\hline CO 55 & 3 & 0,01 & 2500 & 0,004 & 1 & 0,03 & 0,05 \\
\hline Abalovi & 2 & 0,01 & 3125 & 0,005 & 1 & 0,03 & 0,05 \\
\hline Yébéssé djin & 1 & 0,00 & 5000 & 0,007 & 1 & 0,03 & 0,05 \\
\hline Kalé & 2 & 0,01 & 1250 & 0,002 & 1 & 0,03 & 0,04 \\
\hline Adibolo & 2 & 0,01 & 703,125 & 0,001 & 1 & 0,03 & 0,04 \\
\hline Kalipkoé & 1 & 0,00 & 2500 & 0,004 & 1 & 0,03 & 0,04 \\
\hline Demon & 2 & 0,01 & 1250 & 0,002 & 1 & 0,03 & 0,04 \\
\hline Kamme mani & 1 & 0,00 & 625 & 0,001 & 1 & 0,03 & 0,04 \\
\hline Alice & 1 & 0,00 & 0,1 & 0,000 & 1 & 0,03 & 0,04 \\
\hline Kalikpoé & 1 & 0,00 & 0,1 & 0,000 & 1 & 0,03 & 0,04 \\
\hline Gbokalé & 1 & 0,00 & 0,1 & 0,000 & 1 & 0,03 & 0,04 \\
\hline
\end{tabular}

N_Rpdts $=$ Nombre de répondants ;

N_Loc $=$ Nombre de localités ;

$\mathbf{S}^{2}=$ Carré de la surface emblavée par le cultivar ;

FrQRpd (Fréquence des répondants) = Nombre de personnes qui pratiquent la culture du cultivar répertorié/sur le nombre de personnes qui cultivent le piment.

$\mathbf{S}^{2} \mathbf{A c} / \mathbf{S}^{2} \mathbf{T}=$ Superficie emblavée par le cultivar répertorié sur la superficie totale réservée à la culture du piment. FrQLoc (Fréquence des localités) $=$ Nombre de localités dans lequel le cultivar est rencontré sur le nombre de localités enquêtées. IVI = Indice de valeur d'importance. 


\section{DISCUSSION}

La présente étude a permis de recenser 28 contraintes pour la culture du piment dans toutes les zones d'étude contre 14 recensées au Bénin (Orobiyi et al., 2013). De toutes ces contraintes recensées, le déficit hydrique constitue la contrainte la plus citée par les agriculteurs. Cette observation confirme l'impact négatif du manque d'eau sur les cultures de piment (Glodjinon et al., 2019). La construction de retenues d'eau (barrages, forages) reste insuffisante pour résorber le manque d'accès à l'eau des producteurs de piment en particulier mais aussi des populations en général comme en témoigne la réduction de moitié de la quantité d'eau par habitant au Togo entre $1990\left(4000 \mathrm{~m}^{3} /\right.$ an / habitant $)$ et $2014\left(2000 \mathrm{~m}^{3} / \mathrm{an} /\right.$ habitant $)$ (FAO, 2015).

Toutefois, si le déficit hydrique est cité comme la première contrainte majeure dans la production de piment au Togo, c'est plutôt la pauvreté des sols qui est perçue en premier au Bénin (Orobiyi et al., 2013). Par ailleurs, les résultats obtenus ont révélé que la pauvreté des sols vient en deuxième position des contraintes citées. Cette contrainte constitue ainsi un problème majeur commun au Togo et au Bénin. C'est dans ce contexte que plusieurs travaux sont consacrés à la recherche de solutions durables dans la restauration, la fertilisation des sols cultivés au Togo (Toundou et al., 2014) mais aussi l'amélioration de la résistance au déficit hydrique (Bokobana et al., 2019). D'autres contraintes ont également été répertoriées lors de la présente étude.

Ainsi, selon les enquêtés, la faible durée de conservation post-récolte des fruits est très remarquée au niveau des piments du groupe chinense (cas des cultivars Gboyébéssé et Tongoor) qui sont très appréciés des consommateurs grâce à leur qualité organoleptique, contrairement aux piments des groupes frutescens et аппиит non aromatiques mais présentant une durée de conservation relativement longue (cas du cultivar Gobi et Lèguèlèguè). Cette observation est confirmée par une étude au Bénin qui montre que le piment long effilé rouge et le petit piment effilé (respectivement proches des groupes annuum et frutescens) sont les plus séchés. Ils sont ainsi conservables sous forme séchée pendant au moins un an (Yolou, 2019). Les piments verts rond (groupe chinense) ne sont que très peu ou pas conservables par séchage.

Aussi, certaines contraintes répertoriées comme mineures au plan national (faible valeur marchande, manque de marché d'écoulement, excès d'humidité du sol) peuvent constituer, à l'échelle locale, des données non négligeables, et doivent ainsi être prises en compte dans les programmes d'amélioration de la production du piment. En exemple, l'attaque des chenilles constitue une contrainte majeure pour la production du piment dans la région des savanes contrairement aux résultats globaux sur le plan national où elle se classe comme contrainte mineure. Aussi, il existe une relation entre les contraintes répertoriées et les localités prospectées.

Les différentes contraintes liées à la production de piment varient d'une localité à l'autre. Ainsi, la corrélation entre les localités et les contraintes a permis de définir trois groupes de localités dont deux (le groupe II et le groupe III) sont géographiquement homogènes. La pourriture molle citée dans le groupe II (localités de la zone écologique IV) peut s'expliquer par le caractère particulièrement humide de cette zone lié à une pluviométrie élevée et à un couvert végétal dense marqué par la présence de mégaphanérophytes (Atakpama, et al., 2019). Dans le groupe III, qui rassemble des localités plus disparates (Djakperenaag - zone écologique I ; Akparé, Fin digue, Wougba zone écologique IV; Tétékpoé Kopé, Attitongon - zone écologique V), la présence de contraintes abiotiques qui s'opposent (excès d'humidité et déficit hydrique) laisse suggérer un manque de maîtrise de l'utilisation de l'eau (mauvaise conservation en période d'excès et mauvaise utilisation en période de besoin). Cette situation pourrait être justifiée par le manque de main d'œuvre et aurait comme corollaire la forte présence d'attaques d'ordre biotique des cultures 
(anthracnose, flétrissement bactérien, pourriture molle). Le manque de main d'œuvre, par ailleurs, pourrait trouver son origine dans la faible valeur marchande des cultures de piment signalée par les producteurs qui font face à des poches de sécheresse durant lesquelles diverses méthodes sont utilisées.

A la lumière des enquêtes menées, la région centrale connaît les plus longues durées moyennes des poches de sécheresse en pleine saison de pluie, soit environ deux semaines et demie. Durant ces périodes, la culture du piment est stoppée dans $21 \%$ des cas laissant ainsi place à la résurgence des contraintes biotiques (attaque des insectes pour 87,50\% des enquêtés), faute d'entretien des cultures ou de main d'œuvre dédiée disponible. A contrario, la région des savanes pourtant sous un climat soudanien, connaît des poches de sécheresse de plus faibles durées moyennes, soit environ deux semaines. Ces résultats corroborent les données d'Adewi et al. (2010) pour qui la période séparant l'arrêt précoce des pluies de celui tardif est de deux semaines dans la zone soudanienne. Les producteurs de piment se rabattent alors sur des pratiques endogènes consommatrices en eau (irrigation, agriculture sur berge) mais aussi à des techniques empiriques de conservation de l'eau ou de l'humidité (paillage) afin de garantir un meilleur rendement pour les différents cultivars.

Concernant les cultivars répertoriés à travers le pays, les résultats révèlent qu'ils sont au nombre de 34 sous réserve de synonymie et présentent différentes formes. Une caractérisation sur la base des caractères pomologiques du fruit a permis de les regrouper à partir de variables qualitatives en six groupes au seuil de dissimilarité de 1,6 (classification hiérarchique ascendante). Les noms vernaculaires enregistrés peuvent varier d'une région économique à une autre et d'un groupe socio - linguistique à un autre. Ces observations qui sont courantes en nomenclature vernaculaire ont également été rapportées pour de nombreuses cultures dont le piment au Bénin (Orobiyi et al., 2017), mais aussi au Togo pour le taro (Bammite et al.,
2018) et le niébé (Dagnon, 2018). Les caractérisations agro-morphologique, biochimique et moléculaire pourraient alors permettre de clarifier ce problème de synonymie (Adetula et al., 2006 ; Dagnon et al., 2017). Les six groupes de piment dégagés renferment des cultivars dont l'importance est variable.

Ainsi, à chaque cultivar répertorié, un indice de valeur d'importance (IVI) a été attribué. Le cultivar Gboyébéssé est celui qui est le plus prisé. Ce cultivar est rencontré dans toutes les régions du Togo. Parmi les cinq premiers cultivars par indice de valeur d'importance, aucun cultivar introduit ne figure. Cela dénote d'une part que ces derniers ne sont pas encore suffisamment inscrits dans les habitudes paysannes encore moins dans les habitudes des consommateurs. La chaîne de distribution plus restreinte des semences (acquisition exclusivement dans les centres de l'Institut Togolais de la Recherche Agronomique) pourrait être à la base de cette situation. D'autre part, cette observation pourrait trouver son origine dans le souci de conservation des habitudes culinaires et/ou alimentaires des populations.

\section{Conclusion}

Cette étude a permis de recenser les différentes pratiques endogènes liées à la culture du piment au Togo confrontée au déficit hydrique. Les pratiques endogènes adoptées par les agriculteurs pour la plupart font appel aux ressources en eau déjà disponibles. Cette situation à la longue, sans maîtrise des besoins réels en eau du piment pourrait conduire à une utilisation non rationnelle de cette denrée qui se fait de plus en plus rare. La documentation des différents cultivars ainsi que des contraintes dans la culture du piment pourrait servir de base pour un programme d'amélioration variétale et d'augmentation de la productivité. Enfin, une caractérisation plus affinée des cultivars rencontrés, impliquant d'autres caractères pomologiques du fruit (plasticité du tégument, insertion placentaire) pourrait permettre une meilleure discrimination des cultivars. Aussi, du fait que ces caractéristiques observées 
peuvent être influencées par le climat et le sol, une caractérisation biochimique et/ou moléculaire pourrait permettre de dissocier véritablement les différents cultivars de piment au Togo.

\section{CONFLITS D'INTERETS}

Les auteurs déclarent qu'il n'y a aucun conflit d'intérêts relatif à la rédaction de cet article.

\section{CONTRIBUTIONS DES AUTEURS}

Tous les auteurs mentionnés ont contribué à la rédaction du présent manuscrit.

\section{REMERCIEMENTS}

Nos remerciements vont à l'endroit du personnel de l'Institut Togolais de Recerche Agronomique (ITRA), section culture maraichère pour leur précieuse aide.

\section{RÉFÉRENCES}

Adetula AO, Olakojo SA. 2006. Genetic characterization and evaluation of some pepper accessions (Capsicum frutescens L.) : The Nigerian 'Shombo'collections. JAES, 1(3): 273-281. https://www.researchgate.net/publication 1242304814 .

Adewi E, Badameli KMS, Dubreuil V. 2010. Évolution des saisons des pluies potentiellement utiles au Togo de 1950 à 2000. Climatologie, 7 : 89-107. DOI : 10.4267/climatologie.489.

Atakpama W, Amégnaglo KB, Afelu B, Folega F, Batawila K et Akpagana K. 2019. Vertigo - revue électronique en sciences de l'environnement, 19(3). DOI: https://doi.org/10.4000/vertigo.27000.

Badaméli A, Dubreuil V. 2010. Diagnostique du changement climatique au Togo à travers l'évolution de la température entre 1960 et 2010. XXVIII ${ }^{\text {ème }}$ Colloque de l'Association Internationale de Climatologie, Jul 2015, Liège, Belgique. pp. 421 - 426, actes du XXVIII ${ }^{\text {ème }}$ Colloque de l'Association Intrnationale de Climatologie. DOI : halshs-01176808.

Bokobana A, Toundou O, Odah K, Dossou KSS, Tozo K. 2019. Enhancement of proline content and antioxidant enzyme activities induced by application of compost. Int. J. Biol. Chem. Sci., 13(7): 2978-2990.

DOI: https://dx.doi.org/10.4314/ijbcs.v13i7.1.

Dagnon YD, Diop S, Bammite D, Glato K, Gbaguidi AA, Dansi A, Tozo K. 2017. Variabilité agromorphologique des cultivars locaux de niébé [Vigna unguiculata (L.) Walp.] au Togo. Afr. Sci. Rev. Int. Sci. Technol., 13 (4) : 164177. http://www.afriquescience.info.

Dagnon YD. 2018. Gestion paysanne, caractérisation agromorphologique et moléculaire des variétés locales du niébé cultivées au Togo. Thèse de Doctorat, Université de Lomé, 168p.

Dansi, A, Adoukonou-Sagbadja H, Vodouhe R. 2010. Diversity, conservation and related wild species of Fonio millet (Digitaria spp.) in the northwest of Benin. Genet Resour. Crop Ev., 57(6): 827-839.

DOI: https://doi.org/10.1007/s10722-0099522-3.

FAO. 2012. FAOSTAT Database. Food and Agriculture Organization, Roma, Italy. Available online at URL: www.fao.org consulté le 27 juin 2020.

FAO. 2015. FAO Statistical Pocketbook 2015: Food and Agriculture Organization of the United Nations, Rome. $236 \mathrm{p}$.

FAO. 2019. FAOSTAT Database. Food and Agriculture Organization, Roma, Italy. http://www.fao.org/faostat/fr/\#rankings/c ountries_by_commodity consulté le 27 juin 2020 .

Glodjinon NM, Noumavo PA, Adeoti K, Garba K, Kouhounde SH, Ohin BM, Baba-Moussa L. 2019. The technical production, storage and conservation routes of chilli peppers (Capsicum spp.) produced in Benin and constraints impeding the development of the sector. J. Anim. Plant Sci., 42(3): 7279-7295. DOI : https://doi.org/10.35759/JAnmPlSci.v423.

Kouassi KC, Koffi-Névry R. 2012. Evaluation de la connaissance et utilisation des variétés de piment (Capsicum) cultivées 
en Côte d'Ivoire. Int. J. Biol. Chem. Sci., 6(1): $\quad$ 175-185: DOI : http://dx.doi.org/10.4314/ijbcs.v6i1.16.

Krishnareddy M, Rani RU, Kumar KA, Reddy KM, Pappu H. 2008. Capsicum chlorosis virus (Genus Tospovirus) infecting Chili pepper (Capsicum annuum) in India. Plant Dis., 92(10) : 1469-1469. DOI: https://doi.org/10.1094/PDIS-92-101469B.

Kwon SA. 2006. Beyond Resistance! Youth Activism and Community Change. Taylor \& Francis Group; 215-228. https://doi.org/10.4324/9780203961001.

Lemou F. 2008. Le dérèglement pluviométrique et la dynamique de l'occupation et l'exploitation des sols dans le bassin versant de la Kara (NordTogo). Mémoire de DEA, Université de Lomé, $102 \mathrm{p}$.

Loko YL, Dansi A, Dansi M, Vodouhè R, Sanni A. 2013. Indigenous knowledge and management of yam (Dioscorea Cayenensis - Dioscorea Rotundata Complex) pests and diseases in Northern Benin. IJABPT, 4(3): 277-287. https://www.researchgate.net/publication /264429129.

Orobiyi A., Dansi M, Assogba P, Loko L, Vodouhe R, Akouegninou A, Sanni A. 2013. Chili (Capsicum annuum L.) in southern Benin: production constraints, varietal diversity, preference criteria and participatory evaluation. IRJAS, 3(4): 107-120. http://www.interesjournals.org/IRJAS.

Park JE, Yi HB, Kim CG, Jeong SC, Yoon WK, Park KW, Lee BK, Kim HM. 2007. The effect of the anthracnose resistant chili pepper (PepEST gene) on the nontarget insects, green peach aphids (Myzus persicae Sulzer, Homoptera). Korean J. Appl. Entomol., 46 (3): 343-348. DOI : https://doi.org/10.5656/KSAE.2010.49.1. 023.

Reifschneider FJ, Henz GP, Ribeiro CS. 2009. Brazilian capsicums: early history and future prospects. Chron. Hortic., 49(3): 19-21. http://www.ishs.org/chronica.

Simo C, Tchiaze Ifoue AV, Nouage Tayoukam LC, Nsouga Amougou R, Lombeko V, Njonzo-Nzo SA, Djocgoue PF. 2019. Inhibitory effects of aqueous and ethanolic extracts of pepper (Capsicum annuиm) on the development of necrosis caused by Phytophtora megakarya in Theobroma cacao. Int. J. Biol. Chem. Sci., 13(4): 1938-1959. DOI: https://dx.doi.org/10.4314/ijbcs.v13i4.3.

Tilahun S, Paramaguru P, Rajamani K. 2013. Capsaicin and ascorbic acid variability in chilli and paprika cultivars as revealed by HPLC analysis. GJPBG, 1(2) : 85-89. http://www.escijournals.net/JPBG

Toundou O, Tozo K, Feuillade K, Pallier V, Tchegueni S, Dossou KSS. 2014. Effets du compost de déchets sur les propriétés chimiques du sol et la solubilité d'éléments minéraux sous deux régimes hydriques en conditions contrôlées au Togo. Int. J. Biol. Chem. Sci., 8(4): 1917-1926.

DOI : http://dx.doi.org/10.4314/ijbcs.v8i4.51.

Wahyuni Y, Ballester AR, Tikunov Y, de Vos RC, Pelgrom KT, Maharijaya A, Bovy AG. 2013. Metabolomics and molecular marker analysis to explore pepper (Capsicum sp.) biodiversity. Metabolomics, 9(1): 130-144. DOI: 10.1007/s11306-012-0432-6.

Xing Y, Li X, Xu Q, Yun J, Lu Y, Tang Y. 2011. Effects of chitosan coating enriched with cinnamon oil on qualitative properties of sweet pepper (Capsicum annuum L.). Food Chem., 124(4): $1443-1450 . \quad$ DOI: 10.1016/j.foodchem.2010.07.105.

Yolou I. 2019. Risques de pertes post-récoltes et modes endogènes de conservation de produits maraîchers à Parakou (nord du Bénin). Revue Espace Géographique et Société Marocaine, 27 : 19 p. 